\title{
CARACTERIZAÇÃO E UTILIZAÇÃO DA CASCA DA JABUTICABA: UMA REVISÃO DE LITERATURA
}

\author{
Patricia Alvarez Cabanez ${ }^{1}$ \\ Nohora Astrid Velez Carvajal ${ }^{2}$ \\ Lucas Rosa Pereira ${ }^{3}$ \\ Samuel Ferreira da Silva ${ }^{4}$ \\ Lucimara Ribeiro Venial ${ }^{5}$ \\ Carolina de Oliveira Bernardes ${ }^{6}$
}

Resumo: A jabuticaba pode ser consumida in natura ou processada e a sua casca, subproduto do processamento, pode ser reaproveitada devido aos seus constituintes. O objetivo deste trabalho foi estudar a caracterização e utilização da casca da jabuticaba visando o aproveitamento dos seus constituintes para obtenção de outros produtos. O estudo consistiu numa pesquisa realizada através de trabalhos encontrados na base de dados Scielo, além de resumos, livros, teses e dissertações. Constatou-se que a casca da jabuticaba é fonte de vitaminas e minerais, carboidratos, fibras, substâncias antioxidantes e ácidos orgânicos; e pode ser utilizada na fabricação de vários produtos e apresenta vários benefícios para a saúde humana. Conclui-se que a casca da jabuticaba apresenta vários compostos de interesse da indústria farmacêutica e alimentícia e pode ser utilizada no preparo de diferentes produtos alimentícios, em substituição, para o enriquecimento ou como corante natural.

Palavras-chave: Jabuticabeira; Aproveitamento; Resíduo; Fruto; Composição.

\footnotetext{
1 Doutorado em Produção Vegetal/Universidade Federal do Espírito Santo, Brasil. E-mail: patriciacabanez@gmail.com.

2 Doutorado em Produção Vegetal/Universidade Federal do Espírito Santo, Brasil. E-mail: nora_velez@yahoo.com.br.

3 Doutorado em Produção Vegetal/Universidade Federal do Espírito Santo, Brasil. E-mail: lucasrosapereira@hotmail.com.

4 Doutorado em Produção Vegetal/Universidade Federal do Espírito Santo, Brasil. E-mail: samuelfd.silva@yahoo.com.br.

5 Mestrado em Produção Vegetal/Universidade Federal do Espírito Santo, Brasil. E-mail: luci_venial@hotmail.com.

6 Doutorado em Genética e Melhoramento/Universidade Federal do Espírito Santo, Brasil. E-mail: carolinabernardes84@yahoo.com.br.
} 